${ }^{1}$ Pontifical Catholic University of Paraná. Curitiba, PR, Brazil.

${ }^{2}$ Positivo University. Curitiba, PR, Brazil.
Corresponding author:

Prof. Dr. Rodrigo Nunes Rached PUCPR (PPGO)

Rua Imaculada Conceição, 1155 Curitiba, PR, Brazil, 80215-901.

E-mail: r.rached@pucpr.br; ronura@gmail.com

Phone: 55 41-3271-1637

Editor: Dr Altair A. Del Bel Cury

Received: September 1, 2020

Accepted: April 5, 2021

\section{Randomized clinical trial of complete denture cleaning methods in residents of care institutions}

\author{
Ana Paula Sponchiado ${ }^{10}$, Maria Cecília Carlos Lopes \\ Giacomel $^{2}$ (D) Evelise Machado de Souza ${ }^{1}$ (D), \\ Rosimeire Takaki Rosa' ${ }^{1}$ (D, Edvaldo Antonio Ribeiro \\ Rosa $^{1}$ (D), Rodrigo Nunes Rached ${ }^{1, *}$ (i)
}

Aim: To evaluate the efficacy of ultrasonic bath and traditional brushing in the hygiene of complete dentures of dependent residents in long-term care institutions (LTCls). Methods: A randomized cross-over clinical study was conducted in 17 maxillary complete denture wearers living in LTCls. Cleaning protocols were brushing or ultrasonic bath, both applied with neutral liquid soap. Biofilm biomass were estimated by MTT reduction assay and specific microbial load (CFU) of Streptococcus spp., Staphylococcus spp. and Candida spp. were quantified by selective and differential culture media. Results: Ultrasound method showed higher percentage reduction of biofilm biomass and specific microbial loads of Streptococcus spp. compared to brushing $(\mathrm{p}<0.01)$. Reduction of microbial loads of the other microorganisms were not different between cleaning methods ( $p>0.05$ ). Conclusions: The ultrasonic bath proved to be a feasible alternative method for the mechanical cleaning of complete dentures in LTCIs.

Keywords: Dentures. Hygiene. Biofilms. Health services for the aged. Elderly. 


\section{Introduction}

Denture stomatitis affects approximately $65 \%$ of patients with maxillary complete dentures and it is commonly associated to poor oral hygiene'. The condition may be aggravated by trauma, continuous use of the dentures, allergic reaction to the denture base and cleaning products, hyposalivation, inadequate diet and use of antibiotics ${ }^{2,3}$. Candida albicans is the main etiological agent of this condition or associated to other pathogenic and opportunistic microorganisms, such as Streptococcus spp., Staphylococcus spp. and Lactobacillus ${ }^{4-6}$.

Systemic dissemination of microorganisms from oral infections associated with biofilm accumulation in complete dentures may cause bacterial endocarditis, aspiration pneumonia and chronic obstructive pulmonary disease in dependent elderly patients ${ }^{7-9}$. Denture cleaning for biofilm control is essential to reduce the risk of these diseases $^{8}$. Complete dentures can be cleaned using chemical and/or mechanical methods such as hypochlorites, peroxides, enzymes, mouthwashes, brushing, sonic and ultrasonic vibration ${ }^{10,11}$.

Complete dentures wearers in long-term care institutions ( $\mathrm{LTCls)} \mathrm{may} \mathrm{be} \mathrm{unable} \mathrm{to}$ maintain hygiene of their dentures due to illness, dementia or low motor coordination, which makes them dependent on caregivers' support ${ }^{12-15}$. Although these professionals consider oral hygiene important, lack of time, work overload and refusal behavior by patients may difficult its adequate performance ${ }^{16}$.

Brushing alone or associated with soap, toothpaste or abrasives has been the most commonly reported method for cleaning complete dentures $8,11,17$. Ultrasonic bath is an accessible and low-cost cleaning method, especially for old and physically compromised patients ${ }^{18-20}$

The aim of this study was to compare the efficacy of ultrasonic bath and brushing as cleaning methods of complete dentures in LTCI dependent residents. The outcomes evaluated were the percentage changes of biofilm biomass and specific microbial loads after application of the cleaning methods. The work hypotheses were:

1. There would be no difference between cleaning methods for biofilm biomass;

2. There would be no difference between cleaning methods for specific microbial load;

\section{Materials and methods}

The present study was registered in REBEC (RBR-3jj84f) and approved by the Institutional Research Ethics Committee (Protocol Number 2,225,015).

\section{Sample selection}

The sample was composed of individuals selected among residents of two LTCIs institutions located in the metropolitan area of the city of Curitiba (Parana, Brazil). All residents were dependent elderly individuals. Inclusion criteria were 60 years of age or older, complete maxillary edentulism and wear of conventional maxillary complete denture for at least one year. 
Individuals were excluded if: they wore conventional maxillary dentures that were fractured, repaired or relined; were using or had used antibiotics, antifungal agents or corticosteroids for a period of three months prior to the beginning of the research; were using denture adhesives or dentures with excessive calculus; were smokers.

A sample size calculation for a non-inferiority trial of no differences between one treatment (ultrasound) to another (brushing) indicated a sample size of 13 patients for a power of $80 \%{ }^{21}$. The CONSORT 2010 flowchart ${ }^{22}$ (Figure 1) illustrates the selection of study participants, allocation, monitoring and analysis. The institutions and their residents were invited to participate in the research after presentation of the risks, benefits and objectives of the study. The final sample consisted of 17 patients ( 4 men and 13 women) with a mean age of 72.5 years, who provided written informed consent to participate in the study. For the incapable patients, the legally designated representative was contacted, given explanations about the research and asked to provide written informed consent.

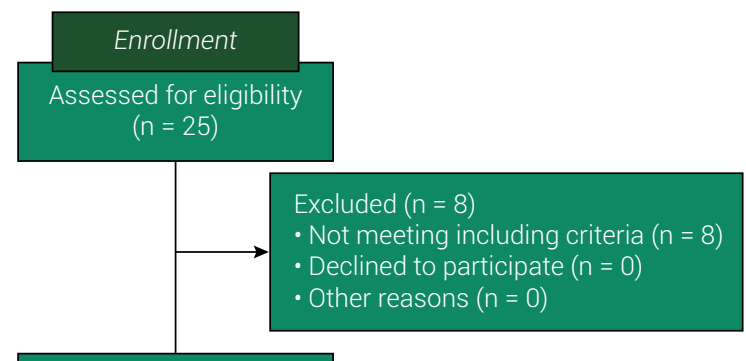

Randomized ( $\mathrm{n}=17)$

Allocation

Allocated to intervention $(n=17)$

Cross-over study: all 17 participants underwent both interventions.

The sequence of interventions was randomized.

- Received allocated intervention $(n=17)$

- Did not receive allocated intervention $(n=0)$

Follow-Up

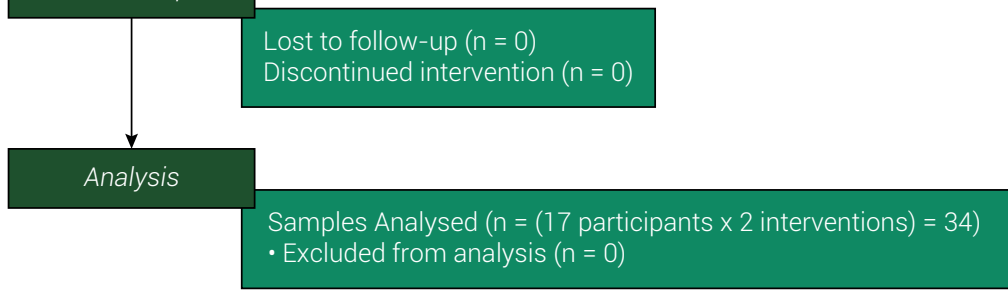

Figure 1. CONSORT 2010 Flow Diagram.

\section{Study design and cleaning methods}

This clinical study was randomized, double-blind, controlled, crossover, clinical study. All dentures were exposed to the two interventions, for five days each step. 
Intervention 1) Manual brushing with neutral dishwashing soap: brushing once a day, at a fixed time, for two minutes ${ }^{20}$ using a soft brush specifically for denture cleaning (Twister, Colgate, São Bernardo do Campo, SP, Brazil) associated with neutral dishwashing soap without glycerin (Ype, Goiania, GO, Brazil).

Intervention 2) Ultrasonic bath with neutral liquid soap solution: The dentures were individually placed into identified and closed glass jars containing $100 \mathrm{~mL}$ of tap water and one drop of neutral dishwashing soap (Ype), which were subjected to ultrasonic vibration (Cristofoli, Campo Mourao, PR, Brazil) at 160 watts once daily, at a fixed time, for 15 minutes $^{19,23}$. The dentures were rinsed in running water and returned to the patient.

One of the researchers (RNR) through a lottery system using wrapped cards placed in a plastic jar containing one of two possible sequences of interventions to be tested performed randomization. During the experimental stage, a reminder poster display was fixed on the bedroom wall of each patient with a daily checklist, according to the randomized sequence of cleaning methods. On the bathroom walls, posters were also fixed to emphasize that denture cleaning was not to be performed by anyone other than the previously calibrated caregiver.

Before the research began, alginate impressions of each maxillary complete denture were taken (Jeltrate, Dentsply, Petropolis, RJ, Brazil) and dentures replicated using acrylic resin (Jet Classic, Sao Paulo, SP, Brazil). This procedure was performed so that the replicates could be digitized with a dental scanner (Ceramill map400, Amann Girrbach, Koblach, Austria) and the images were then analyzed using the Solid Edge v20 software (Siemens PLM, Plano, USA) to calculate the total area of the dentures $\left(\mathrm{cm}^{2}\right)$, which comprised both the external surface of the prosthesis (palate and dental arch) and the internal and retention area.

With the purpose of starting the research with all dentures at the same level of hygiene, they were immersed in $4.2 \%$ acetic acid solution for 10 min and then brushed with $2 \%$ chlorhexidine digluconate (FGM, Joinville, SC, Brazil) for two min ${ }^{24}$. The dentures were washed with running water and returned to the patients. They wore the dentures normally for two days, and these were cleaned by brushing once a day. On the third day of the research, possible dietary debris were eliminated by using an indirect vortex of tap water for $30 \mathrm{~s}$, with each denture being placed in a plastic receptacle with a volume of $500 \mathrm{~mL}$. Subsequently, the dentures were immersed in $100 \mathrm{~mL}$ of sterile saline solution. The sets were taken to the ultrasonic vessel for $15 \mathrm{~min}$ and the suspensions obtained were considered baseline in the microbiological analyses.

After this collection, the dentures were again disinfected by immersion in $0.12 \%$ chlorhexidine digluconate (Periogard $B$, Colgate-Palmolive, Sao Paulo, SP, Brazil) for 15 min, followed by brushing with 2\% chlorhexidine gel (Maquira, Maringa, PR, Brazil) for 2 min and returned to the patients, whose dentures were then exposed to the study procedures.

Each of the cleaning methods was implemented for five consecutive days, and after a washout period of one week between each stage, the steps previously mentioned were repeated for all patients prior to beginning with the second intervention, so that all subjects were submitted to both cleaning methods. After apply- 
ing each of the methods, the dentures were immersed in identified sterilized glass jars containing $100 \mathrm{~mL}$ of saline solution. The sets were taken to the ultrasonic vessel for $15 \mathrm{~min}$ and the suspensions obtained were considered "treatment" in the microbiological analyses.

The "baseline" and "treatment" suspensions were sonicated (2840D, Odontobrás, Ribeirao Preto, SP, Brazil) for 20 min for maximum biofilm disintegration. Aliquots of $500 \mu \mathrm{L}$ were collected for quantification of the specific microbial load. The remaining suspension was used for estimating biofilm biomass by the reduction test of 3-(4, 5-dimethylthiazol-2-yl)-2, 5-diphenyltetrazolium bromide (MTT; Sigma-Aldrich M5655, St Louis, MO, USA) ${ }^{25}$.

\section{Specific Microbial Load Quantification}

The following dilution protocol was determined after a pilot trial with a patient's complete denture.

Five hundred microliters aliquots of the suspensions were combined with $500 \mu \mathrm{L}$ of distilled water and vortexed (AP56, Phoenix, Araraquara, SP, Brazil) at 1500 rpm for $30 \mathrm{~s}$. Aliquots of $100 \mu \mathrm{L}$ were taken and combined with $900 \mu \mathrm{L}$ of distilled water and again vortexed at $1500 \mathrm{rpm}$ for $30 \mathrm{~s}$.

One hundred microliter-aliquots of the above-mentioned diluted suspensions were plated in selective and differential culture media Chromocult (Merck, Germany), Manitol Salt Agar (NewProv, Brazil) and Sabouraud Agar Dextrose (NewProv, Brazil). These media allow the presumptive quantification of Streptococcus spp. (after confirmation by Gram staining), Staphylococcus spp. and Candida spp., respectively. Plates containing media for bacterial quantification were incubated at $37^{\circ} \mathrm{C}$ and plates with yeast media were incubated at $28^{\circ} \mathrm{C}$ (Orion-502, Fanem, SP, Brazil). All incubations were conducted for $72 \mathrm{~h}$ in a normoxic atmosphere.

After the incubation periods, the specific microbial load of the dentures was determined by counting the number of colonies formed (CFU = colony forming units) that presented phenotypes indicating the studied genera, followed by correction for dilution. The specific microbial density was obtained by dividing the numerical CFU values by the area of the dentures $\left(\mathrm{cm}^{2}\right)$.

\section{Estimation of biofilm biomass by MTT reduction assay}

The sonicated suspensions were centrifuged at 4500×g (BE-4004, Bioeng, Curitiba, PR, Brazil) for $10 \mathrm{~min}$. The supernatants were removed and $1.5 \mathrm{~mL}$ of sterile water was added. After resuspension and transfer to microtubes, the suspensions were centrifuged at 10,000×g (Eppendorf, Hamburg, Germany) for 1 min. After removal of the supernatant, $500 \mu \mathrm{L}$ of $1 \mathrm{mg} / \mathrm{mL}^{-1} \mathrm{MTT}$ was added and the microtubes were incubated at $37^{\circ} \mathrm{C}$ for $3 \mathrm{~h}$. After every one-hour time interval the microtubes were vortexed at $1500 \mathrm{rpm}$ for $30 \mathrm{~s}$. At the end of the incubation, $500 \mu \mathrm{L}$ of distilled water was added to the microtubes, which were agitated and again centrifuged for $3 \mathrm{~min}$. The supernatants were removed and $200 \mu \mathrm{L}$ of isopropyl alcohol was added to the sediments. After agitating for 30 seconds, the contents were separated at 10,000×g for $1 \mathrm{~min}$. One hundred microliter-aliquots of the supernatants were collected and transferred 
to wells of a flat-bottom microplate reader. The optical densities of the formazan crystals formed were measured in a TP-Reader (Thermoplate, GO, Brazil) at $540 \mathrm{~nm}$. The optical density values were divided by the area of the respective dentures. The final results were expressed as $\mathrm{OD}_{540} / \mathrm{cm}^{2}$.

Estimation of biofilm biomass by MTT reduction assay and specific microbial load were performed by a blinded investigator (APS).

\section{Statistical analysis}

Statistical analyses were made with the Statistical Package for Social Science 24.0 (SPSS Inc, Chicago, IL, USA). Data were tested for normality by the Kolmogorov-Smirnov test with Lilliefors significance correlation, and Shapiro-Wilk test. Comparisons between cleaning methods were made using the Mann-Whitney U-test. Comparisons between the percentage change from baseline to treatment of biofilm biomass and specific microbial load count were done with Wilcoxon signed test. All tests were performed at a significance level of $5 \%$.

\section{Results}

The means and standard deviation of percentage change of biofilm biomass for brushing and ultrasound methods are presented in Table 1. Ultrasound method showed a significantly higher percentage change of biofilm biomass compared to brushing $(p<0.05)$ (Table 1). Means and 95\% confidence intervals of biofilm biomass are shown in Figure 2.

Table 1. Means and standard deviations of percentage change of biofilm biomass.

\begin{tabular}{lll}
\hline \multicolumn{2}{c}{ Mean (士standard deviation) } \\
\hline Brushing & $-23.62 \%( \pm 51.12 \%)$ & $p=0.0004857$ \\
\hline Ultrasound & $-70.77 \%( \pm 16.95 \%)$ & \\
\hline
\end{tabular}

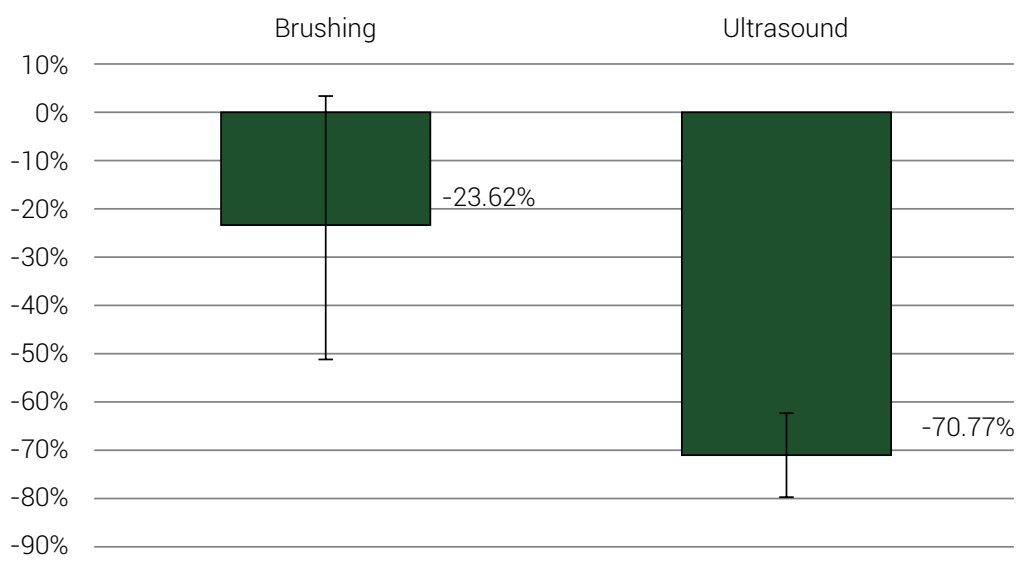

Figure 2. Means and 95\% confidence intervals of percentage change of biofilm biomass. 
The means and standard deviation of percentage change of specific microbial loads of Streptococcus spp., Staphylococcus spp. and Candida spp. are shown in Table 2. Streptococcus spp. was the only group that showed significant reduction in the count of microorganisms in both methods. Staphylococcus spp. and Candida spp. showed significant reduction in the percentage of microorganisms only in ultrasound method. Means and 95\% confidence intervals of specific microbial loads are shown in Figure 3.

Table 2. Means and standard deviations of percentage change of specific microbial load for Streptococcus spp., Staphylococcus spp. and Candida spp. according to cleaning method.

\begin{tabular}{|c|c|c|c|}
\hline & Cleaning Method & $\begin{array}{c}\text { Mean } \\
\text { ( } \pm \text { standard deviation) }\end{array}$ & \\
\hline \multirow{2}{*}{ Streptococcus spp. } & Brushing & $-23.39 \%( \pm 45.95 \%)$ & \multirow{2}{*}{$p=0.009$} \\
\hline & Ultrasound & $-42.51 \%( \pm 37.70 \%)$ & \\
\hline \multirow{2}{*}{ Staphylococcus spp. } & Brushing & $-18.99 \%( \pm 42.81 \%)$ & \multirow{2}{*}{$p=0.094$} \\
\hline & Ultrasound & $-36.54 \%( \pm 44.07 \%)$ & \\
\hline \multirow{2}{*}{ Candida spp. } & Brushing & $-16.27 \%( \pm 35.37 \%)$ & \multirow{2}{*}{$p=0.239$} \\
\hline & Ultrasound & $-24.07 \%( \pm 37.05 \%)$ & \\
\hline
\end{tabular}

$\mathrm{UFC} / \mathrm{cm}^{2}$

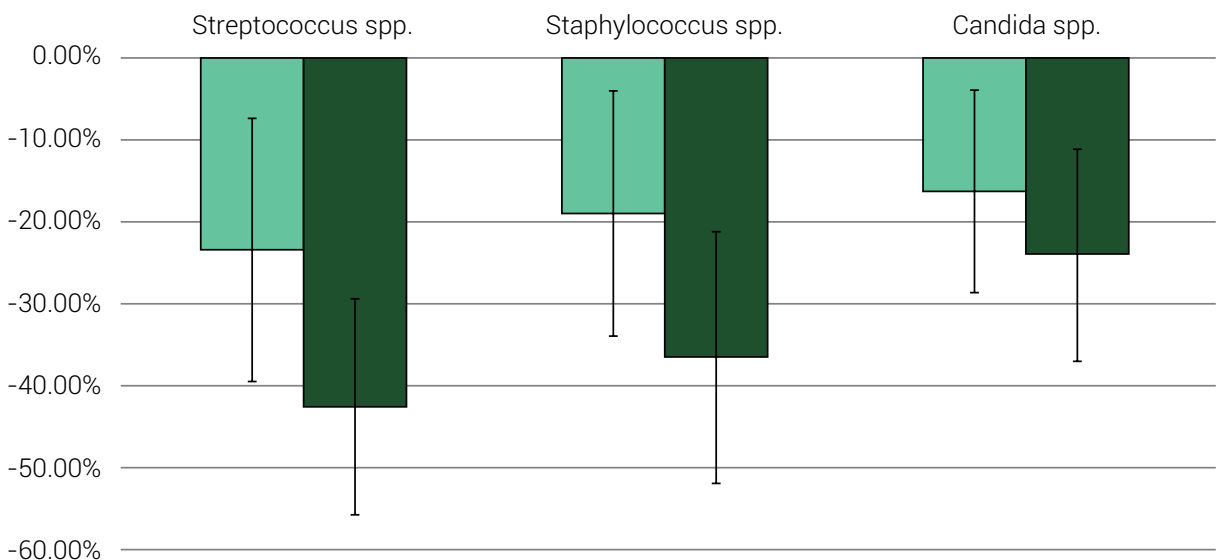

$-60.00 \%$

$$
\text { Brushing } \quad \square \text { Ultrasound }
$$

Figure 3. Mean and 95\% confidence intervals of percentage change of microbial loads.

\section{Discussion}

The current study compared the efficacy of the mechanical cleaning methods brushing and ultrasonic bath, on denture biofilm mass and specific microbial loads. The first null hypothesis was rejected as the positive impact of ultrasonic cleaning on biofilm mass was superior to brushing. The second null hypothesis was confirmed for the Streptococcus spp. group, which showed significant changes on the percentage of specific microbial load in both methods, whereas it was rejected for Staphylococ- 
cus spp. and Candida spp. groups, which had significant change on the percentage of specific microbial load only in ultrasound method.

The current study compared the efficacy of the mechanical brushing cleaning and ultrasonic bath methods on denture biofilm mass and specific microbial loads. The first work hypothesis was rejected since the positive effect of ultrasonic cleaning on biofilm mass was superior to brushing. The second work hypothesis was rejected since the Streptococcus spp. group showed significant changes on the percentage of specific microbial load in both methods.

The choice of estimating biofilm biomass by the MTT reduction essay was shown to be adequate, since it is a widely used method in different areas of study ${ }^{25,26}$, and is applicable for quantifying the microbial content covering dental materials ${ }^{27}$. However, MTT reduction essay in complete denture cleaning methods is innovative. The method of scores based on biofilm staining, although frequently cited in the literature, is limited and not very accurate $20,24,28-30$. The Polymerase Chain Reaction (PCR), another widely used test for biofilm evaluation in complete dentures $24,31,32$, does not allow discrimination of living cells from dead cells, since it is based on the indistinct amplification of DNA segments, and may promote erroneous estimation of the microbial load ${ }^{24}$. On the other hand, the estimation of microbial biomass by the quantification of formazan formed from the MTT reduction is more reliable for estimating the load of living cells ${ }^{26}$. In the present study, the ultrasound method showed to be more efficient in the reduction of the percentage compared to brushing.

As for the effect of cleaning methods on the percentage of Streptococcus ssp. microorganism count, a statistically significant reduction was shown for brushing and ultrasound groups. This reduction has also been previously demonstrated ${ }^{23}$, however, the ultrasonic bath was associated with effervescent tablets and applied only once at the end of the research.

In the current study, the counts of Staphylococcus spp. and Candida spp. were similarly reduced by the two cleaning methods. Another study also reported a decrease in the levels of Candida spp. with ultrasound associated to effervescent tablets ${ }^{19}$. On the other hand, studies have not observed reduction in Candida spp., neither with the method of brushing nor ultrasound, associated or not with effervescent tablets ${ }^{23,24}$.

A previous study has suggested that $S$. mutans from the biofilm of complete dentures create a glucan barrier that could limit the exposure of other species to chemical cleaning methods, including Candida spp ${ }^{33}$. Reduced counts of Candida spp. were found when bactericidal and/or fungicidal solutions were associated with ultrasonic bath $^{34}$. Based on these results, it is possible to propose that this association could be effective, since the mechanical cleaning with ultrasound is capable of destabilizing the superficial layers of biofilm on dentures, that contain glucans produced by Streptococcus spp. This disruption would expose the Candida spp. of deeper layers of the biofilm to the action of chemicals ${ }^{33-35}$.

The results of the current study comply with these assumptions, since significant reduction in the microbial load was only shown for Streptococcus spp., both in the ultrasound and brushing groups. These microorganisms are found more superficially in the biofilm layers and, thus, may be more easily removed. On the other hand, Can- 
dida spp. and Staphylococcus spp., are found in deeper biofilm layers, being positively influenced by the ultrasound method.

Ubiquitous microorganisms, such as Candida spp., are present in healthy patients or in those with prosthetic stomatitis. In addition, Candida albicans is found in the prostheses of patients with pathological changes ${ }^{1,6}$. Counts of Candida spp. in multi-species biofilm of complete dentures are difficult to reduce even when mechanical cleaning and effervescent tablets are associated ${ }^{23,33,36}$. In the current study, only the ultrasound was able to reduce the microbial load of Candida spp.

Staphylococcus spp. are related to pneumonia that affects many elderly residents in $\mathrm{LTCls}^{7}$. In the current study, they were significantly reduced in concentration when the ultrasound method was applied to the complete dentures. Therefore, it is expected that the method will result in minimizing a possible etiological factor for respiratory diseases related to these microorganisms.

Although brushing with water and toothpaste is the most commonly cleaning method used by complete denture wearers ${ }^{8,11,17}$, elderly patients may experience a decline in hand dexterity that affects their capacity to brush the dentures effectively ${ }^{19}$. Ultrasonic cleaning is not a commonly used technique for denture in nursing homes, probably due to lack of information of caregivers and residents, but could be effective in an institutional home that provides care for the elderly with physical or cognitive deficiencies ${ }^{19,37,38}$. The effectiveness of ultrasonic cleaning is not dependent on the number of caregivers of the institution, as opposed to the brushing method. The method makes it possible to clean more than one denture at the same time. Other advantages are easy handling of the equipment and low risk of occupational infection to caregivers. In the current study, the ultrasonic bath proved to be efficient in all the analyzed parameters: capability of significantly reducing the biofilm biomass as shown in the MTT assay, as well as of reducing the microbial load for all microorganisms studied groups.

Chemical disinfection is effective when associated with biofilm on denture surfaces ${ }^{30,39}$. There is a considerable variety of chemicals available for denture cleaning; however, the difficulty in manipulating and the elevated cost of some of these products limits their adoption in LTCls. As an example, effervescent tablets have demonstrated to be effective for the cleaning of dentures, however, they have an elevated cost, making it difficult to establish their use in underprivileged institutions ${ }^{20,40,41}$. An ideal cleaning agent for complete dentures should remove organic and inorganic materials, be non-toxic, cost-effective, easy to handle, and reduce biofilm ${ }^{39}$.

As an example, dishwashing liquid soap, a conventional cleaning product, has proven to present emulsifying action that degrades biofilms. Nevertheless, ultrasonic cleaning has demonstrated to be more effective when associated to chemical agents with these characteristics ${ }^{23,35}$. The chemical action results from protein solubilization and consequent reduction in microbial adhesion on complete denture surfaces ${ }^{40,42}$. The advantage over effervescent tablets is centered on non-abrasiveness, low cost and accessibility ${ }^{43}$.

Finally, it is important to note that, as proposed herein, the prostheses should be somehow stored individually during ultrasonic bath conduction, avoiding possible cross-contamination between residents. 
In conclusion, the ultrasound method was more effective than brushing in reducing the biofilm biomass and the count of Streptococcus spp. The reduction percentage of the specific count of the microorganisms Staphylococcus spp. and Candida spp microorganisms did not differ between the two cleaning methods.

The ultrasonic bath proved to be a feasible alternative method for mechanical cleaning of complete dentures in LTCIs.

\section{Acknowledgments}

The authors thank the elderlies of the Long Term Care Institutions and their families who collaborated and supported the project. We also thank the caregivers and all staff of these Institutions, and a special thanks to the caregiver Eva Alice Cavalli who have taken an active participation during the experimental phase.

\section{References}

1. Budtz-Jorgensen E, Stenderup A, Grabowski M. An epidemiologic study of yeasts in elderly denture wearers. Community Dent Oral Epidemiol. 1975 May;3(3):115-9. doi: 10.1111/j.1600-0528.1975.tb00291.x.

2. Salerno C, Pascale M, Contaldo M, Esposito V, Busciolano M, Milillo L, et al. Candidaassociated denture stomatitis. Med Oral Patol Oral Cir Bucal. 2011 Mar;16(2):e139-43. doi: 10.4317/medoral.16.e139.

3. Oksala E. Factors predisposing to oral yeast infections. Acta Odontol Scand. 1990 Feb;48(1):71-4. doi: 10.3109/00016359009012736.

4. Baena-Monroy T, Moreno-Maldonado V, Franco-Martínez F, Aldape-Barrios B, Quindós G, Sánchez-Vargas LO. Candida albicans, Staphylococcus aureus and Streptococcus mutans colonization in patients wearing dental prosthesis. Med Oral Patol Oral Cir Bucal. 2005;10 Suppl 1:E27-39.

5. Barnabé W, De Mendonça Neto T, Pimenta FC, Pegoraro LF, Scolaro JM. Efficacy of sodium hypochlorite and coconut soap used as disinfecting agents in the reduction of denture stomatitis, Streptococcus mutans and Candida albicans. J Oral Rehabil. 2004 May;31(5):453-9.

6. O'Donnell LE, Robertson D, Nile CJ, Cross LJ, Riggio M, Sherriff A, et al. The oral microbiome of denture wearers is influenced by levels of natural dentition. PLoS One. 2015 Sep ;10(9):e0137717. doi: 10.1371/journal.pone.0137717.

7. Mylotte JM. Nursing Home-Associated Pneumonia. Clin Geriatr Med. 2007 Aug;23(3):553-65, vi-vii. doi: 10.1016/j.cger.2007.02.003..

8. Coulthwaite L, Verran J. Potential pathogenic aspects of denture plaque. Br J Biomed Sci. 2007;64(4):180-9. doi: 10.1080/09674845.2007.11732784.

9. Scannapieco FA, Shay K. Oral health disparities in older adults: oral bacteria, inflammation, and aspiration pneumonia. Dent Clin North Am. 2014 Oct;58(4):771-82. doi: 10.1016/j.cden.2014.06.005.

10. Paranhos HFO, Silva-Lovato CH, Souza RF, Cruz PC, Freitas KM, Peracini A. Effects of mechanical and chemical methods on denture biofilm accumulation. J Oral Rehabil. 2007 Aug;34(8):606-12. doi: 10.1111/j.1365-2842.2007.01753.x..

11. Nikawa $\mathrm{H}, \mathrm{Hamada} \mathrm{T}$, Yamashiro $\mathrm{H}$, Kumagai $\mathrm{H}$. A review of in vitro and in vivo methods to evaluate the efficacy of denture cleansers. Int J Prosthodont. 1999;12(2):153-9. 
12. De Visschere L, De Baat C, Schols JMGA, Deschepper E, Vanobbergen J. Evaluation of the implementation of an "oral hygiene protocol" in nursing homes: A 5-year longitudinal study. Community Dent Oral Epidemiol. 2011 Oct;39(5):416-25. doi: 10.1111/j.1600-0528.2011.00610.x.

13. Zenthöfer A, Dieke R, Dieke A, Wege K-C, Rammelsberg P, Hassel AJ. Improving oral hygiene in the long-term care of the elderly-a RCT. Community Dent Oral Epidemiol. 2013 Jun;41(3):261-8. doi: $10.1111 /$ cdoe. 12007 .

14. Gil-Montoya JA, de Mello ALF, Cardenas CB, Lopez IG. Oral Health Protocol for the Dependent Institutionalized Elderly. Geriatr Nurs (Minneap). 2006;27(2):95-101. doi: 10.1016/j.gerinurse.2005.12.003.

15. Gornitsky M, ParadisI I, Landaverde G, Malo A-M, Velly AM. A clinical and microbiological evaluation of denture cleansers for geriatric patients in long-term care institutions. J Can Dent Assoc. 2002 Jan;68(1):39-45.

16. Gibney J, Wright C, Sharma A, Naganathan V. Nurses' knowledge, attitudes, and current practice of daily oral hygiene care to patients on acute aged care wards in two Australian hospitals. Spec Care Dent. 2015;35(6):285-93. doi: 10.1111/scd.12131.

17. Jagger DC, Harrison A. Denture cleansing--the best approach. Br Dent J. 1995 Jun;178(11):413-7. doi: 10.1038/sj.bdj.4808788.

18. Kawasaki K, Kamikawa Y, Sugihara K. In vitro and in vivo removal of oral Candida from the denture base. Gerodontology. 2016 Jun;33(2):247-52. doi: 10.1111/ger.12149.

19. Nishi Y, Seto K, Kamashita Y, Kaji A, Kurono A, Nagaoka E. Survival of microorganisms on complete dentures following ultrasonic cleaning combined with immersion in peroxide-based cleanser solution. Gerodontology. 2014 Sep;31(3):202-9. doi: 10.1111/ger.12027.

20. Cruz PC, Andrade IM De, Peracini A, Souza-Gugelmin MCM De, Silva-Lovato CH, Souza RF De, et al. The effectiveness of chemical denture cleansers and ultrasonic device in biofilm removal from complete dentures. J Appl Oral Sci. 2011;19(6):668-73. doi: 10.1590/s1678-77572011000600021.

21. Slade GD, Akinkugbe AA, Sanders AE. Projections of U.S. edentulism prevalence following 5 decades of decline. J Dent Res. 2014 Oct;93(10):959-65. doi: 10.1177/0022034514546165.

22. Schulz KF, Altman DG, Moher D. CONSORT 2010 statement: Updated guidelines for reporting parallel group randomised trials. PLoS Med. 2010 Mar 24;7(3):e1000251. doi: 10.1371/journal.pmed.1000251.

23. De Andrade IM, Cruz PC, Da Silva CHL, De Souza RF, Paranhos HFO, Candido RC, et al. Effervescent tablets and ultrasonic devices against Candida and mutans streptococci in denture biofilm. Gerodontology. 2011 Dec;28(4):264-70. doi: 10.1111/j.1741-2358.2010.00378.x.

24. Duyck J, Vandamme K, Krausch-Hofmann S, Boon L, Keersmaecker K De, Jalon E, et al. Impact of denture cleaning method and overnight storage condition on denture biofilm mass and composition: A cross-over randomized clinical trial. PLoS One. 2016 Jan;11(1):e0145837. doi: 10.1371/journal.pone.0145837.

25. Mosmann T. Rapid colorimetric assay for cellular growth and survival: Application to proliferation and cytotoxicity assays. J Immunol Methods. 1983 Dec;65(1-2):55-63. doi: 10.1016/0022-1759(83)90303-4.

26. van Meerloo J, Kaspers GJL, Cloos J. Cell sensitivity assays: the MTT assay. Methods Mol Biol. 2011;731:237-45. doi: 10.1007/978-1-61779-080-5_20.

27. Brambilla E, Gagliani M, Ionescu A, Fadini L, García-Godoy F. The influence of light-curing time on the bacterial colonization of resin composite surfaces. Dent Mater. 2009 Sep;25(9):1067-72. doi: 10.1016/j.dental.2009.02.012. 
28. De Souza RF, Paranhos HFO, Lovato da Silva CH, Abu-Naba'a L, Fedorowicz Z, Gurgan CA. Interventions for cleaning dentures in adults. Cochrane Database Syst Rev. 2009 Oct;(4):CD007395. doi: 10.1002/14651858.CD007395.pub2.

29. Paranhos $\mathrm{H}$ de FO, Silva $\mathrm{CHL}$ da. Comparative study of methods for the quantification of biofilm on complete dentures. Braz Oral Res. 2004 Jul-Sep;18(3):215-23. doi: 10.1590/s1806-83242004000300007.

30. Paranhos H de FO, da Silva CHL, Venezian GC, Macedo LD, de Souza RF. Distribution of biofilm on internal and external surfaces of upper complete dentures: the effect of hygiene instruction. Gerodontology. 2007 Sep;24(3):162-8. doi: 10.1111/j.1741-2358.2007.00177.x.

31. Campos MS, Marchini L, Bernardes LAS, Paulino LC, Nobrega FG. Biofilm microbial communities of denture stomatitis. Oral Microbiol Immunol. 2008 Oct;23(5):419-24. doi: 10.1111/j.1399-302X.2008.00445.x.

32. Morino T, Ookawa K, Haruta N, Hagiwara Y, Seki M. Effects of professional oral health care on elderly: randomized trial. Int J Dent Hyg. 2014 Nov;12(4):291-7. doi: 10.1111/idh.12068.

33. Drake D, Wells J, Ettinger R. Efficacy of denture cleansing agents in an in vitro bacteria-yeast colonization model. Int J Prosthodont. 1992;5(3):214-20.

34. Hashiguchi M, Nishi Y, Kanie T, Ban S, Nagaoka E. Bactericidal efficacy of glycine-type amphoteric surfactant as a denture cleaner and its influence on properties of denture base resins. Dent Mater J. 2009 May;28(3):307-14. doi: 10.4012/dmj.28.307.

35. Budtz-Jørgensen E. Materials and methods for cleaning dentures. J Prosthet Dent. 1979 Dec;42(6):619-23. doi: 10.1016/0022-3913(79)90190-2.

36. Ferreira MÁF, Pereira-Cenci T, Rodrigues de Vasconcelos LM, Rodrigues-Garcia RCM, Del Bel Cury AA. Efficacy of denture cleansers on denture liners contaminated with Candida species. Clin Oral Investig. 2009 Jun;13(2):237-42. doi: 10.1007/s00784-008-0220-x.

37. Palenik CJ, Miller $\mathrm{CH}$. In vitro testing of three denture-cleaning systems. J Prosthet Dent. 1984 Jun;51(6):751-4. doi: 10.1016/0022-3913(84)90369-x.

38. Shay K. Denture hygiene: A review and update. J Contemp Dental Pract. 2000 Feb;1(2):28-41.

39. Silva-Lovato $\mathrm{CH}$, Wever B, Adriaens E, Paranhos HFO, Watanabe E, Pisani MX, et al. Clinical and antimicrobial efficacy of NitrAdine TM-based disinfecting cleaning tablets in complete denture wearers. J Appl Oral Sci. 2010 Dec;18(6):560-5. doi: 10.1590/s1678-7757201000060000.

40. Moore TC, Smith DE, Kenny GE. Sanitization of dentures by several denture hygiene methods. J Prosthet Dent. 1984 Aug;52(2):158-63.

41. H Nikawa, T Yamamoto, T Hamada, S Sadamori SA. Cleansing Efficacy of commercial denture cleansers: Ability to Reduce Candida albicans biofilm Activity. Int J Prosthodont. 1995 NovDec;8(6):527-34.

42. Landa AS, van de Belt-Gritter B, van der Mei HC, Busscher HJ. Recalcitrance of Streptococcus mutans biofilms towards detergent-stimulated detachment. Eur J Oral Sci. 1999 Aug;107(4):236-43. doi: 10.1046/j.0909-8836.1999.eos107402.x.

43. Salles AES, Macedo LD, Fernandes RAG, Silva-Lovato CH, Paranhos HFO. Comparative analysis of biofilm levels in complete upper and lower dentures after brushing associated with specific denture paste and neutral soap. Gerodontology. 2007 Dec;24(4):217-23. 